\title{
Planning for Landing Site Selection in the Aerial Supply Delivery *
}

\author{
Aleksandr Kushleyev ${ }^{\dagger}$ ， Brian MacAllister ${ }^{\dagger}$ ，Maxim Likhachev ${ }^{\ddagger}$ \\ University of Pennsylvania ${ }^{\dagger}$, Carnegie Mellon University
}

\section{Introduction}

Autonomous aerial robots can sometimes be tasked to deliver supplies to various remote locations. Their high speed and operating altitude offer advantages over ground robots. However, UAVs need to take off and land, often requiring special equipment and algorithms for finding suitable landing locations close to the goal. This paper studies the problem of planning a sequence of flight and sensing actions required to find and land at the best landing site using a minimum amount of resources.

Figure 1 demonstrates a sample scenario: the UAV needs to deliver a payload to a location marked $G O A L$. Based on prior information, it identifies 7 possible landing sites. In addition to site-specific landing costs, each location has an availability status, which is unknown at the start, but is probabillistic in nature (modeling uncertainty in the environment). The task of the planner is to decide where the helicopter should fly, sense, and potentially land in order to minimize the expected sum of the flight time, landing, and the cost of delivering the supplies from the landing location to the goal.

To compute this policy, one could use assumptive planning techniques (Koenig and Smirnov; Nourbakhsh and Genesereth 1996) - such a planner would assume that all possible landing sites are valid. The plan would be to fly to the site that minimizes the objective function, land there if it is acceptable, otherwise, fly to the next one. While fast to compute, these approaches do not minimize the expected cost and can result in a waste of resources.

In this paper, we present an approach that does minimize the expected cost. Unknown land site status makes this problem generally hard to solve, however, we show that it exhibits clear preference on uncertainty, making it solvable via a recently proposed approach PPCP (Probabilistic Planning with Clear Preferences) (Likhachev and Stentz 2006). PPCP solves large scale planning under uncertainty problems in anytime fashion while providing rigorous theoretical guarantees on the solution quality. Our analysis of simulation results and real helicopter demonstrations show that our approach can solve problems with up to 15 landing sites in un-

${ }^{*}$ This research was partially sponsored by the DARPA grant N10AP20011 and TATRC contract W81XWH-07-C-0043 Copyright (c) 2011, Association for the Advancement of Artificial Intelligence (www.aaai.org). All rights reserved.

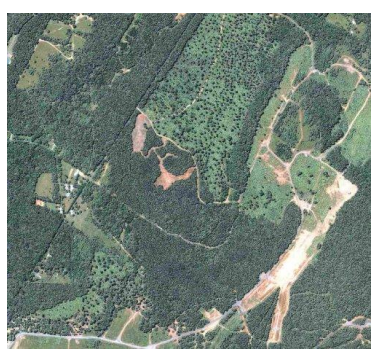

(a) satellite image

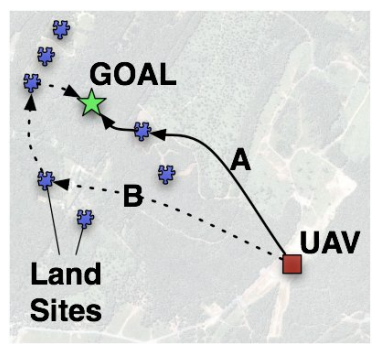

(b) start, goal, candidate land sites
Figure 1: Landing site selection problem. (a) shows a sample satellite image that can be used for extracting candidate landing sites shown in (b). Trajectories A and B are only two out of $O\left(c^{n}\right)$ possible paths with no loops for $n$ sites.

der a second as well as is feasible to run on a real unmanned system. A more detailed version of this paper can be found in (Kushleyev, MacAllister, and Likhachev 2011).

\section{Planning Landing Site Selection}

PPCP Algorithm. The PPCP algorithm is well-suited for planning in the environments that are only partially-known. The algorithm assumes that the true environment itself is fully deterministic and can be modeled as a graph. The goal of the planner is to construct a policy that reaches any goal state while minimizing the expected cost of execution.

The main requirement of the PPCP algorithm is that the problem must exhibit clear preferences on unknown information. This means that for any stochastic transition, it must be always clear which value of the missing variable results in the best outcome. Mathematically, for any unknown variable $h$, the preferred value exists and is equal to $b$ if for any state $X$ in which $h$ is unknown and action $a$, which reveals the value of $h$, there exists a (best) successor state $X^{\prime}$ which has $h=b$ and satisfies the requirement

$$
X^{\prime}=\operatorname{argmin}_{Y \in \operatorname{succ}(X, a)} c(S(X), a, S(Y))+v^{\star}(Y)
$$

where $S(X)$ represents the part of $X$ that is always observable.

PPCP exploits the existence of clear preferences to scale up to very large problems. In particular, PPCP constructs and refines a plan by running a series of low-dimensional deterministic A*-like searches ( (Likhachev and Stentz 2006)). 


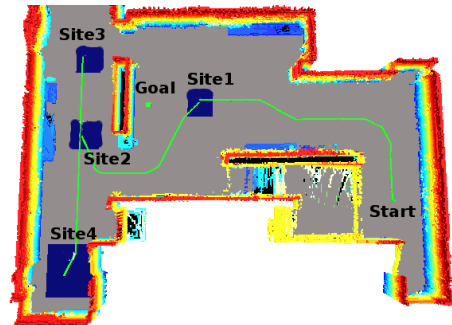

(a) 3D map, landsites, PPCP policy

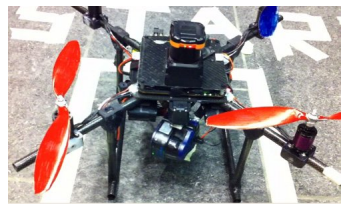

(b) Ready to take off

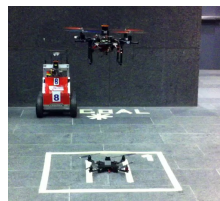

(c) Sensing Site 1

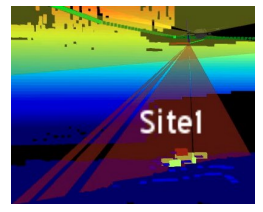

(d) Sensing Site1 (map)

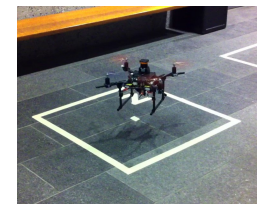

(e) Landing at Site2

Figure 2: 3D map and stills from an autonomous run

Application of PPCP to Landing Site Selection. In the context of our problem, we claim that the preferred outcome of a sensing action always exists. Consider the following for the proof. Let $X_{g}$ and $X_{b}$ be the good and bad successors of any state $Y$ after performing a sensing action. Let $v^{\star}\left(X_{b}\right)$ be the cost of executing the optimal policy at $X_{b}$. Similarly, we define $v^{\star}\left(X_{g}\right)$ for $X_{g}$. Also, let $A\left(X_{b}\right)$ and $A\left(X_{g}\right)$ be the sets of possible further actions at the corresponding states. According to our formulation, $A\left(X_{g}\right)=\left\{A\left(X_{b}\right)\right.$, land $\}$. This is true since if the outcome of sensing action is good, the further action set is augmented by the ability to land. Therefore, the robot at $X_{g}$ still has an option of following $X_{b}$ 's optimal policy and $v^{\star}\left(X_{g}\right) \leq v^{\star}\left(X_{b}\right)$, making $X_{g}$ the preferred outcome. This is a consequence of the fact that knowing that a landing site is good does not invalidate $X_{b}$ 's (or, in fact, any other state's) optimal policy.

The PPCP algorithm exhibits memoryless property - it does not remember the preferred outcomes of stochastic actions. This enables it to efficiently solve large-scale problems with uncertainty without traversing the whole belief state space, however the solution may be suboptimal if the true optimal policy relies on remembering preferred outcomes. We can never know whether the optimal policy requires memory, but our implementation allows to vary the number of remembered preferred outcomes (memory slots), trading off with execution speed.

Theoretical Properties. The expected cost of the PPCP policy after full convergence, is upper-bounded by the optimal policy found by any memoryless planner, assuming that PPCP assumptions hold. This means that while being very efficient, our planner cannot be beaten (cost-wise) by any memoryless planner. If one or more memory slots are used in the PPCP algorithm, the guarantees are even better. For $\mathrm{k}$ memory slots, the upper bound drops to the optimal policy found by any planner that can remember the last k good landing sites. Formally, our planner provides the following guarantee on the quality of solution:

Theorem 1 The policy returned by our memoryless planner is guaranteed to be cost-minimal (optimal) if there exists an optimal policy that does not require the helicopter to sense a landing site without landing at it when it is found to be a good landing site.

\section{Experimental Analysis}

We have implemented the PPCP planner, adapted to this problem, and tested it in simulations for its ability to solve large problems (up to 15 sites). The table in Figure 3 summarizes these results. We also compared our PPCP implemen-

\begin{tabular}{cccc}
$\begin{array}{c}\text { Number of } \\
\text { sites }\end{array}$ & $\begin{array}{c}0 \text { mem slot } \\
(\mathrm{msec})\end{array}$ & $\begin{array}{c}1 \text { mem slot } \\
(\mathrm{msec})\end{array}$ & $\begin{array}{c}\text { Belief state } \\
\text { space size }\end{array}$ \\
\hline 5 & $0.6 \pm 0.3$ & $1.7 \pm 0.9$ & $4.1 \times 10^{3}$ \\
10 & $32 \pm 36$ & $205 \pm 290$ & $1.9 \times 10^{6}$ \\
15 & $560 \pm 1000$ & $15000 \pm 11000$ & $6.7 \times 10^{8}$
\end{tabular}

Figure 3: Average planning times for memoryless PPCP and with one memory slot. The size of the full belief state space is given for reference - PPCP planner finds the solution by computing a tiny fraction of this space.

\begin{tabular}{ccc} 
\# PPCP (expected) & PPCP (actual) & Assumptive Planner \\
\hline 1839.05 & 1839.16 & 1947.25
\end{tabular}

Figure 4: Summary of the results over 2500 runs with 10 landing sites, comparing the expected cost of the PPCP policy, actual cost of traversing PPCP policy, and actual cost of following an assumptive planner

tation with an assumptive planner, which assumes that all the landing locations are good and just replans when sensed otherwise. The comparison was done by simulating the randomly generated site probabilities and computing actual execution costs of both policies. Figure 4 shows the results after running 2500 planning scenarios. PPCP policies were consistently better in execution by about $5 \%$.

In addition, we have tested the planner on a real quadrotor platform, equipped with a dual core processor and LIDAR sensors, shown in Figure 2(b). All the processing was performed on-board : perception, landing planning, motion planning and low-level control. Planning for four landing sites took $0.2 \mathrm{msec}$. Figure 2(a) shows the planned policy and Figure 2(b-e) presents still photographs from the fully autonomous flight according to the policy.

\section{References}

Koenig, S., and Smirnov, Y. Sensor-based planning with the freespace assumption. In Proceedings of the IEEE International Conference on Robotics and Automation (ICRA).

Kushleyev, A.; MacAllister, B.; and Likhachev, M. 2011. Planning for landing site selection in the aerial supply delivery. In Under submission.

Likhachev, M., and Stentz, A. 2006. PPCP: Efficient probabilistic planning with clear preferences in partially-known environments. In Proceedings of the National Conference on Artificial Intelligence (AAAI).

Nourbakhsh, I., and Genesereth, M. 1996. Assumptive planning and execution: a simple, working robot architecture. Autonomous Robots Journal 3(1):49-67. 